\title{
A PESQUISA EM ENFERMAGEM NO BRASIL: RETROSPECTIVA HISTORICA
}

\author{
Maria Jacyra de Campos Nogueira*
}

NOGUEIRA, M. J. de C. A pesquisa em enfermagem no Brasil: retrospectiva histórica. Rev. Esc. Enf. USP, São Paulo, 16(1):17-26, 1982.

$O$ trabalho descreve os tipos $e$ as linhas das pesquisas em enfermagem realizadas no Brasil entre as décadas de 50 a 80 . Predominaram, nesse periodo, pesquisas sobre assistência, seguidas de pesquisas sobre educaçāo. Conclui que as pesquisas estiveram voltadas, provavelmente, para as necessidades do momento histórico do Pais.

\section{INTRODUÇĀO}

"Ao exemplo do passado em que as enfermeiras não se davam conta de coisas importantes que já estavam acontecendo com o grupo profissional, infere-se que hoje podemos deixar de valorizar aspectos sutis da estrutura de nosso grupo profissional que, no futuro, vão se delinear com mais clareza" (DOURADO) ${ }^{10}$.

Atendendo ao pedido da Comissão de Aperfeiçoamento do Pessoal de Nível Superior (CAPES) e do Conselho Nacional de Desenvolvimento Tecnológico (CNPq) para apresentar, em 1982, um trabalho no "II Seminário de Pesquisa em Enfermagem" em Brasilia, fizemos um levantamento retrospectivo sobre as pesquisas na área de enfermagem, desde seus primórdios, até os dias atuais.

Este trabalho teve como objetivo conhecer as principais linhas de pesquisa em enfermagem a fim de fornecer subsídios para a avaliação de suas tendências gerais e sua contribuição social.

A preocupação com a produção científica da enfermagem brasileira já foi objeto de dois trabalhos mais recentes que consideramos muito importantes, a saber: "A produção do conhecimento na Pós-Graduação em Enfermagem no Brasil" 2 e "Produção científica em enfermagem no Brasil: 1960-1979" "16.

\section{METODOLOGIA}

Foram consultadas, para o presente estudo, as principais fontes brasileiras de referência de pesquisa em enfermagem, a saber:

\footnotetext{
* Professor Assistente Doutor do Lepartamento de Enfermagem Médico-Cirúrgica da Escola de Enfermagem da USP - disciplinas Saúde da Comunidade e Enfermagem Preventiva e Comunitária. (Enfermeira).
} 


\section{- Periódicos de enfermagem}

Anais de Enfermagem

(1946 - 1954)

Revista Brasileira de Enfermagem

(1954 - 1980)

Revista da Escola de Enfermagem da USP

(1967 - 1981)

Enfermagem Atual

Enfoque

(1978 - 1981)

(1972 - 1981)

Revista Paulista de Enfermagem

$(1981)$

Revista Gaucha de Enfermagem

(1976 - 1980)

Enfermagem em Novas Dimensões

(1975 - 1979)

- Dados do Centro de Pesquisa em Enfermagem (CEPEn) da Associação Brasileira de Enfermagem $(\mathrm{ABEn})^{4}$

- Documentário sobre a Associação Brasileira de Enfermagem (ABEn) ${ }^{8}$

- Relatórios dos Cursos de Pós-Graduação em Enfermagem (19801981) que incluem pesquisas em andamento (CAPES).

- Banco de teses ${ }^{6}$

Os critérios para a seleção dos trabalhos a serem analisados foram:

- terem sido publicados em território nacional;

- terem sido realizados por enfermeiros;

- terem sido realizados por outros profissionais mas cujo tema se refere à enfermagem.

De acordo com o critério do autor, esses trabalhos de pesquisa foram classificados nas seguintes linhas de pesquisa discriminadas a seguir:

Necessidades do Paciente: pesquisas referentes à identificação de conhecimentos, atitudes, comportamentos e necessidades sentidas em relação à saúde e assistência de enfermagem prestada a grupos de pacientes e à comunidade.

Avaliação da Assistência: pesquisas cujo objetivo foi identificar ou avaliar a problemática da assistência à saúde ou assistência de enfermagem que estava sendo prestada a indivíduos, família e comunidade.

Necessidades do Pessoal de Enfermagem: pesquisas referentes à identificação de conhecimentos, atitudes, comportamentos e necessidades sentidas, do pessoal de enfermagem (profissional e auxiliar), em relação ao seu trabalho.

Metodologia de Enfermagem: pesquisas, geralmente experimentais, que propunham métodos, técnicas, rumos, subsídios ou enfoques diferentes a serem dados à assistência de enfermagem a grupos de pacientes, familias e comunidade e pesquisas bibliográficas ou monografias, com as mesmas finalidades. 
Profissão de Enfermagem: trabalhos de pesquisa sobre o estudo de papéis, funções e atribuições da enfermagem na equipe de saúde, na equipe de enfermagem e nos serviços de saúde, bem como a identificação de problemas relativos à ética e ao exercício profissional.

Ensino de Enfermagem: pesquisas para identificar problemas ou propor novos métodos e técnicas de ensino de enfermagem aos niveis de graduação, pós-graduação e de formação de pessoal auxiliar e de educação continuada.

Epidemiológicas e Biológicas: pesquisas que englobaram o conhecimento e a descrição de eventos biológicos ou de agravos à saúde em grupos de individuos.

\section{RESULTADOS E COMENTÁRIOS}

A pesquisa em enfermagem, no Brasil, como a própria evolução da profissão, provavelmente sofreu as influências naturais da história social do País. Teve início há cêrca de trinta anos.

Podemos considerar como primeiro trabalho documentado de investigação, o "Censo de 1950", embora se considere como pesquisa relevante de enfermagem, o "Levantamento de Recursos e Necessidades de Enfermagem, no Brasil", realizado posteriormente. Foi aquele realizado pela Seção de Organização Sanitária do Departamento Nacional de Saúde (DNS) do Ministério da Educação e Saúde (MES). Esse levantamento foi feito em colaboração com a ABEn, então denominada Associação Brasileira de Enfermeiras Diplomadas (ABED), com a Divisão de Ensino do Serviço Especial de Saúde Pública e com a Diretoria Geral do DNS ${ }^{8,10}$.

O relatório final desse primeiro trabalho ${ }^{8}$, de âmbito nacional, divulgado no IV Congresso Nacional de Enfermagem, em 1950, recomendou ao MES a criação da Seção de Enfermagem no DNS e a realização periódica de novos censos.

Este primeiro trabalho motivou e subsidiou, mais tarde, o importante "Levantamento de Recursos e Necessidades de Enfermagem", já citado, realizado entre 1956 e 1958, pela Associação Brasileira de Enfermagem e financiada pela Fundação Rockefeller ${ }^{8}$.

Podem ser considerados ainda, como trabalhos pioneiros e que esboçam a tentativa de pesquisa no campo da enfermagem, embora não utilizassem a mesma metodologia precisa das atuais pesquisas, sendo alguns, apenas relato de experiências, os seguintes, realizados na década de cincoenta: "Recrutamento e seleção de candidatos a cursos de enfermagem e de auxiliares de enfermagem" "; "Coordenação de tempo e movimento" "; "Aplicação do psicodiagnóstico miocinético na seleção de candidatas às escolas de enfermagem e de auxiliares de enfermagem" "5; "Programa de recrutamento e seleção de candidatos às escolas de enfermagem e de auxiliares de enfermagem da Bahia" ${ }^{13}$; "O serviço 
de enfermagem em quatro hospitais especializados para tuberculose" 11 e "Altas a pedido formuladas por doentes internados em sanatórios de tuberculose antes de ser efetuada a cura" ${ }^{14}$.

Como pode ser verificado (Tabela 1 ), as primeiras investigações das enfermeiras brasileiras e que se encontram documentadas, tratam de temas que, até hoje, constituem preocupações ou linhas de pesquisa em enfermagem, a saber: ensino de enfermagem, profissão de enfermagem, necessidades sentidas pelos pacientes, avaliação da assistência de enfermagem que estava sendo prestada e estudo de métodos para melhorar a qualidade do cuidado e o rendimento das atividades da enfermeira.

$\mathrm{Na}$ década de sessenta, as pesquisas continuaram a seguir as mesmas linhas (Tabela 1) sendo que houve um acréscimo de trabalhos sobre a profissão de enfermagem que chegaram a representar $44,4 \%$ em relação aos demais.

A motivação para a realização de trabalhos sobre essa temática, nas décadas de cincoenta e sessenta, parece ter sido influenciada pela própria época evolutiva da enfermagem latino-americana ${ }^{15}$. As décadas de cincoenta e sessenta representam o final do ciclo de desenvolvimento do País e, consequientemente, das práticas de saúde e da enfermagem, periodo que vai de 1930 a 1960 e que foi caracterizado pelo surgimento e fortalecimento do processo de industrialização. As práticas de saúde estavam dirigidas para a proteção da mão de obra e o aumento da produtividade, situação que pressionou a indústria hospitalar, com um crescimento vertiginoso entre 1940 e $1960^{15}$.

A prática de enfermagem nessas décadas, influenciada por esta situação, teve as seguintes caracteristicas ${ }^{15}$ :

- houve um reconhecimento da importância dos serviços de enfermagem tecnicamente competentes e duplicação do número de escolas de enfermagem, além do aumento da escolaridade exigida para ingresso, com conseqüente melhoria da qualificação profissional;

- a prática de enfermagem voltada predominantemente para a área hospitalar fez com que houvesse necessidade da formação e treinamento de pessoal auxiliar.

No Brasil, na década de sessenta, com a reforma universitária e, principalmente com a passagem da enfermagem para nivel superior (Leis $\mathrm{n}^{\circ} 775 / 49$ e $\left.2.995 / 56\right)^{7}$ e conseqüente implantação de cursos de pósgraduação na região sudeste, a pesquisa em enfermagem foi impulsionada.

Encontramos (Tabela 2) entre as documentadas, de 1950 a 1969, uma média de 1,4 pesquisas por ano. De 1970 a 1981 a média das documentadas, incluindo as em andamento, de acorco com os relatórios dos cursos de pós-graduação em enfermagem foi de 42,0 . 


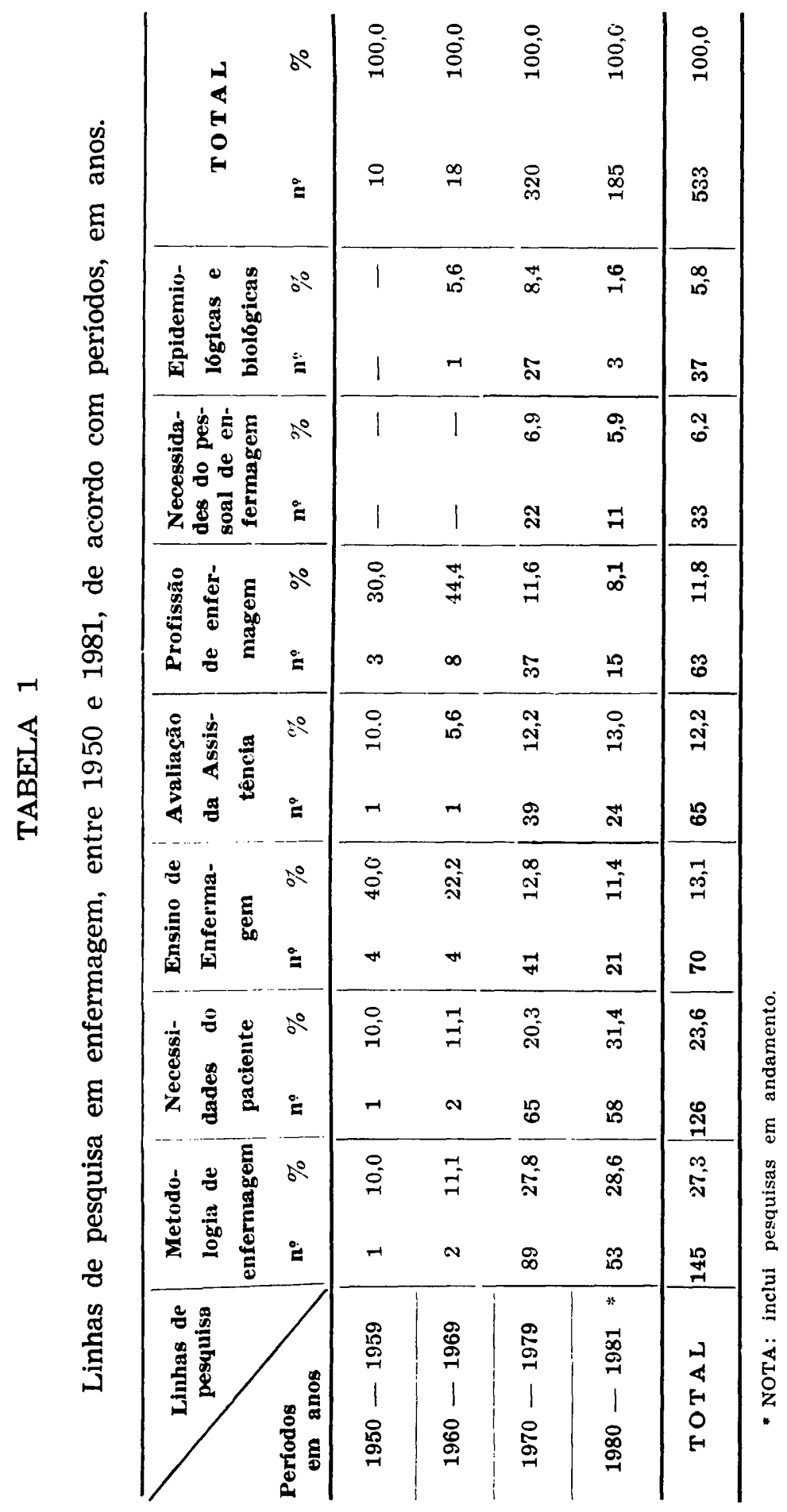




\section{TABELA 2}

Volume e média, por ano, de pesquisas em enfermagem entre 1950 e 1981 de acordo com periodos, em anos.

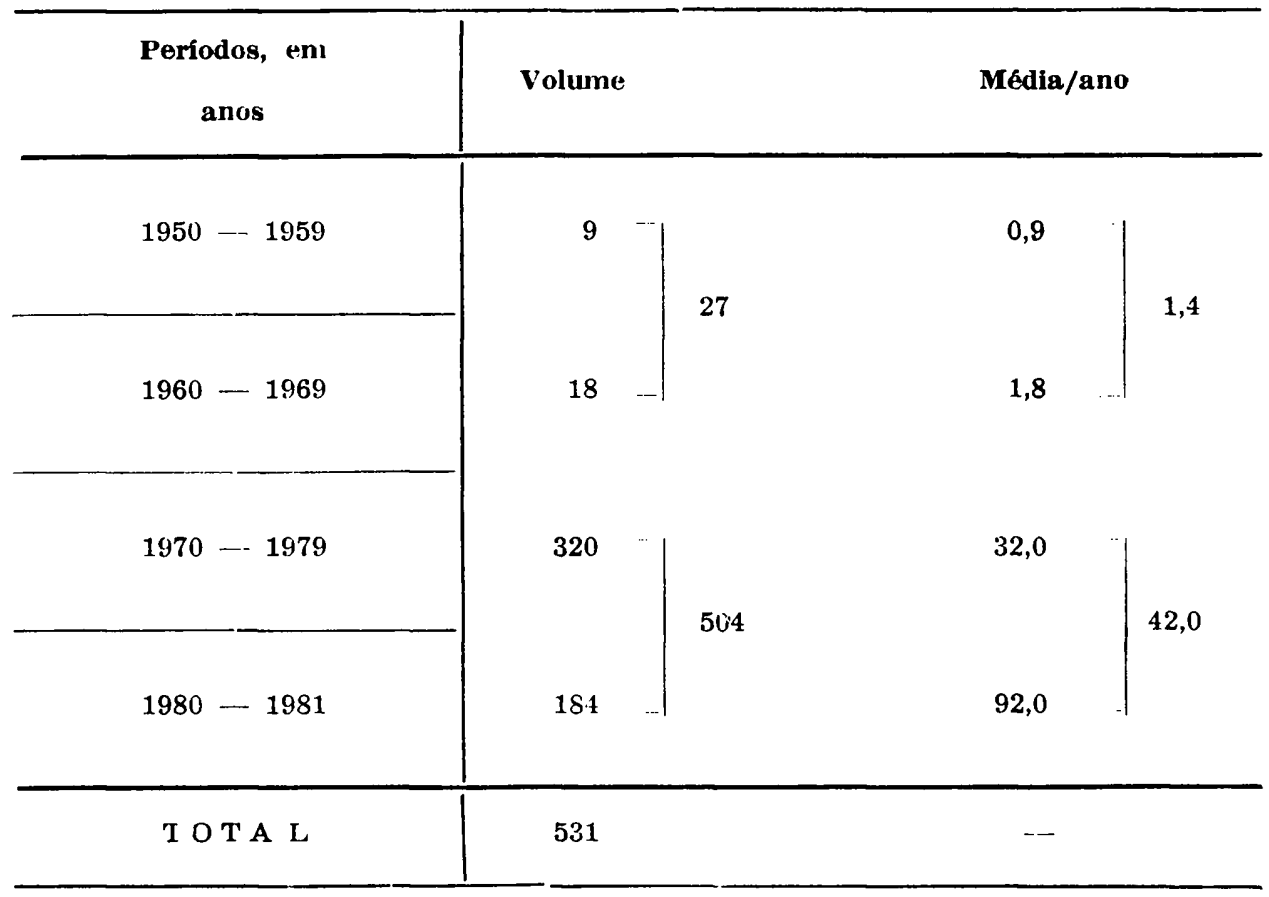

Por outro lado, alguns fatos na evolução da enfermagem latinoamericana ${ }^{15}$ vieram contribuir, também, para esse "posicionamento" da enfermagem brasileira, em relação à busca de princípios norteadores. As práticas de saúde começaram a ser dirigidas, não só para os aspectos curativos, como também para os preventivos, pois os gastos públicos com os serviços de saúde estavam muito voltados para o primeiro aspecto. Essas mudanças fundamentais foram oficializadas por ocasião da Reunião dos Ministros de Saúde do Continente que estabeleceram as metas do Plano Decenal de Saúde para as Américas (1972-1981). Esse plano teve reflexos mais ou menos na estruturação dos serviços de saúde, na política de formação de recursos humanos e na criação de novas metodologias de trabalho.

Embora a prática de enfermagem, na época, ainda continuasse a ser centrada no hospital, surge a tendência a mudar o seu enfoque administrativo por um enfoque clínico. Aparecem cursos de especialização em enfermagem materno-infantil, enfermagem médico-cirúrgica e enfermagem psiquiátrica, onde se discutem os novos papéis da profissão. Há um incremento da educação superior de enfermagem e do 
nümero de escolds le enfermagem. A enrermagem começa também, a se esforçar para assumir a liderança nos programas de extensão de cobertura (assistência primária) e a preparar-se para assumir um papel nessa área ${ }^{15}$.

Verifica-se que, talvez, por influências dessa situação da prática de enfermagem (tabela 1), houve um aumento não só da pesquisa nessa área mas, também, o surgimento de novas linhas de investigação, ou, ainda, o incremento de outras. Começam a ser estudadas, por meio de pesquisas exploratórias, os conhecimentos, atitudes, comportamentos e necessidades sentidas pelo pessoal de enfermagem (enfermeiras e pessoal auxiliar) em relação ao seu trabalho. São incrementadas as pesquisas epidemiológicas e biológicas, em grande parte, talvez, devido aos primeiros orientadores das primeiras dissertações e teses das enfermeiras, terem sido da área médica; outra causa talvez tenha sido a necessidade de um conhecimento maior da normalidade dos fatos vitais e da ocorrência dos agravos à saúde nas populaçöes, já que o enfoque a ser dado, pela prática de enfermagem na época, era o preventivo e o comunitário.

As investigações sobre a metodologia de enfermagem representaram a maior porcentagem da década de setenta $(27,8 \%)$, em relação às outras linhas de pesquisa. Elas foram, em parte, influenciadas, a nosso ver, pelo ensino de pós-graduação em enfermagem e, principalmente, pelas disciplinas que abordavam as novas teorias, os novos papéis da enfermeira e o novo processo de planejar e assistir aos pacientes, em países onde a enfermagem é mais desenvolvida. Elas referem-se, principalmente, à procura de novos métodos, técnicas, rumos e subsídios para a melhoria da assistência de enfermagem. São pesquisas experimentais, principalmente, que propõem sistematização para as ações e atividades de enfermagem nos serviços não só hospitalares, como também, de saúde pública.

Os estudos relativos às necessidades do paciente apareceram como segunda linha de pesquisa mais focalizada na época $(20,3 \%)$. Demonstram a preocupação das enfermeiras em querer prestar a assistência baseada nas necessidades sentidas pela sua clientela e não somente naquelas sentidas pela equipe de saúde com a finalidade, também, de melhorar a qualidade dessa mesma assistência. São principalmente estudos que levantam as necessidades, os conhecimentos, as atitudes e os comportamentos de grupos de pacientes e da comunidade.

Nos dois últimos anos do período estudado (1980 e 1981) a maior porcentagem $(31,4 \%)$ de pesquisas em enfermagem refere-se à identificação das necessidades do paciente ou clientela da enfermagem e, em seguida $(28,6 \%)$, à busca de métodos e técnicas para a melhoria da assistência.

Resumidamente, de 1956 até 1981, as pesquisas em enfermagem foram, na sua maioria $(80,5 \%)$, mais descritivas e exploratórias, do que 
experimentais $(19,5 \%)$ e seguiram, em ordem decrescente, as seguntes linhas (Tabela 1):

- metodologia de enfermagem;

- necessidades do paciente;

- ensino de enfermagem;

- avaliação da assistência;

- profissão de enfermagem;

- necessidades do pessoal de enfermagem;

- epidemiológicas e biológicas.

Segundo um estudo realizado para avaliar a produção de dissertações e teses em enfermagem ${ }^{2}$, as pesquisas nessa área se concentram primordialmente, também, na busca de melhoria da assistência isto é, nos aspectos internos da prática, enquanto prática técnica.

Um trabalho ${ }^{16}$ que teve a finalidade de avaliar a produção científica em enfermagem entre 1960 e 1979 e que incluiu outros trabalhos e não só pesquisas, mostrou que a área predominante foi a de educação ou ensino de enfermagem, na década de cincoenta e de assistência, na década seguinte. Mostrou, ainda, que os trabalhos classificados na área de assistência vêm apresentando processo numérico ascendente, enquanto as publicações, da área de educação vêm sofrendo decréscimo quantitativo.

Nos trabalhos da área de assistência observa-se que predominaram os realizados no campo hospitalar até a década de sessenta quando, então, começaram a ser incrementados os relativos à área comunitária.

Muitas dificuldades e limitações para a realização dessas pesquisas têm surgido. Durante o "I Seminário Nacional de Pesquisa em Enfermagem" 3, realizado em 1979 e sob o patrocínio da ABEn e do CNPq, os grupos de participantes levantaram dentre outras, as seguintes:

- as agências financiadoras, de modo geral, enfatizam mais a pesquisa pura, isto é, aquela que cria alguma coisa nova ou a feita em laboratórios mas, a pesquisa em enfermagem é, geralmente operacional sendo o laboratório a própria clientela;

- as escolas de enfermagem ainda não definiram a sua política em relação à pesquisa em enfermagem, principalmente aquela relativa à aquisição e distribuição de recursos humanos e a locação de recursos materiais;

- os centros de pesquisa em enfermagem, no Brasil, são em número muito reduzido; 
- as enfermeiras que se interessam em pesquisa, estão sobrecarregadas com outras atividades;

- as enfermeiras têm, ainda, conhecimentos restritos sobre as teorias de enfermagem, ciências humanas e correlatas, metodologia de pesquisa e política de saúde do País;

- as instituições de apoio à pesquisa desconhecem a necessidade de pesquisa em enfermagem;

- o intercâmbio entre profissionais que fazem pesquisa em enfermagem é inexistente e as pesquisas realizadas têm pouca divulgação;

- as linhas prioritárias para a pesquisa em enfermagem, não estão, ainda, definidas.

Um ponto crucial levantado pelos autores que estudaram a produção científica da pós-graduação em enfermagem ${ }^{2}$ no País, e que merece estudos foi em relação ao não aproveitamento, na prática, dos resultados das pesquisas em enfermagem realizadas, até hoje, no Brasil. Segundo eles, nem sempre a enfermagem tem podido introduzir mudanças que foram mostradas por meio de pesquisa, para melhorar a assistência ao paciente. Fica aqui o desafio para verificar a amplitude desse fato e a luta para a posterior mudança da realidade se ela se mostrar dessa maneira.

\section{CONCLUSŌES}

A pesquisa em enfermagem no Brasil embora com dificuldades para a sua realização, teve seu início há trinta anos e parece que esteve influenciada pela evolução da própria enfermagem no País. A fase de maior incremento foi, sem dúvida, na década de setenta, devido, principalmente, à influência dos cursos de pós-graduação.

As linhas de pesquisa principais, desde 1950 até 1981 relacionam-se, principalmente, aos aspectos metodológicos da assistência, seguido dos aspectos relativos ao ensino de enfermagem. Essas linhas parecem, a nosso ver, estar correspondendo, prioritariamente às necessidades do momento histórico ou sendo influenciadas pelas práticas de saúde decorrentes da evolução social do País.

Neste momento, entretanto, após a análise retrospectiva da nossa história profissional, várias dúvidas e perguntas nos vêm à mente. Em primeiro lugar, será que todas essas pesquisas, principalmente as de cunho experimental, e as suas propostas foram, realmente, colocadas em prática ou operacionalizadas? Até que ponto as enfermeiras têm tido, nos serviços de saúde e nas escolas de enfermagem, oportunidade de aplicar seus novos conhecimentos decorrentes dos resultados de uma investigação científica? Serão, realmente, as linhas seguidas aquelas prioritárias para a enfermagem brasileira? Fica aqui, o desafio para que, com novas investigações, possamos esclarecer estas questões... 
NOGUEIRA, M. J. de C. Historical retrospective of nursing research in Brazil. Rev. Esc. Enf. USP, São Paulo, 16(1):17-26, 1982.

Types and lines of nursing research in Brazil, from 1950 to 1980 , are described. The majority of studies are on nursing care, followed by nursing education. The Author concludes that probably these studies were an answer to the needs of the country.

\section{REFERENCIAS BIBLIOGRAFFICAS}

1. AlCANTARA, G. Recrutamento e seleção de candidatos a cursos de enfermagem e de auxiliares de enfermagem. Anais Enf., Rio de Janeiro, 7(4):330-41, nov. 1954.

2. ALMEIDA, M. C. P. de et alii. A produção do conhecimento na pós-graduação em enfermagem no Brasil. In: CONGRESSO BRASILEIRO DE ENFERMAGEM, 33\%, Manaus, 2-8, 1981. 21p.

3. ASSOCIAÇAO BRASILEIRA DE ENFERMAGEM. Relatório do Seminário Nacional de Pesquisa em Enfermagem. Ribeirão Preto, CEPEn, 1979. 148p.

4. —_ Informaçōes sobre pesquisas e pesquisadores em enfermagen. Ribeirão Preto, CEPEn, 1979-1980. v. 1-2.

5. ALVIN, E. F. \& CAVALCANTI, B. Aplicação do psicodiagnóstico miocinético na seleção de candidatas a escolas ae enfermagem e de auxiliares de enfermagem. Anais Enf., Rio de Janeiro, $7(4): 341-59$, nov. 1954.

6. BRASIL. Ministério da Educação e Cultura. Catálogo do banco de teses. Brasilia, 19761979. v. $1-4$.

7. BRASIL. Ministério da Saúde. Enfermagem: legislậ̃o e assuntos corrolatos. 3 . ed. Rio de Janeiro, 1974, v. 2.

8. Carvalho, A. C. de Associacão Brasileira de Enfermagem: 1926-1976. Documentário. Brasilia, ABEn, 1976. 514p.

9. Chiarello, F. \& CARValho, A. C. de Coordenação de tempo e movimento. Anais Enf., Rio de Janeiro, 6(3):184-94, ágo, 1953.

10. DOURADO, H.G. Levantamento censitário. Anais Enf., Rio de Janeiro, 3(4):170-3, nov. 1950.

11. $O$ serviço de enfermagem em quatro hospitais especializados para tuberculose. Anais Enf., Rio de Janeiro, $7(4): 394-401$, nov. 1954.

12. - A enfermeira e a institucionalização da profissão e de seu novo papel profissional. Rev. Bras. Enf, Brasilia, 31(3):293-303, jul./set. 1978.

13. OLIVEIRA, M.I.R. Programa de recrutamento e seleção de candidatos as escolas de enfermagem e de auxiliares de enfermagem na Bahia. Anais Enf., Rio de Janeiro, $7(4): 360-3$, nov. 1954 .

14. PONTES, C. As altas a pedido formuladas por doentes internados $\epsilon m$ sanatórios de tuberculose antes de ser efetuada a cura. Anais Enf., Rio de Janeiro, 7(4):417-27, nov. 1954 .

15. VERDERESE, R. $T$. Análisis de la enfermería en America Latina. Educ. med. salud. Washington, 13(4):315-40, 1979.

16. VIEIRA, T. T. Proçução cientifica em enfermagem no Brasil: 1960-1979. Salvador, 1980. (Tese- Professor Titular. Universidade Federal da Bahia). 200p. 